\title{
Impact of Metabolic-Syndrome Risk Factors on the Absenteeism of Health Workers from a Brazilian University Hospital
}

\author{
Almerinda Luedy ${ }^{1}$, Danile Leal Barreto ${ }^{1}$, Hugo Costa-Ribeiro Júnior ${ }^{1,2 *}$ \\ ${ }^{1}$ Complexo Hospitalar Universitário Professor Edgard Santos, Universidade Federal da Bahia, Salvador, Brazil \\ ${ }^{2}$ Federal University of Bahia School of Medicine, Salvador, Brazil \\ Email: *hugoribeiroj r@gmail.com
}

How to cite this paper: Luedy, A., Barreto, D.L. and Júnior, H.C.-R. (2018) Impact of Metabolic-Syndrome Risk Factors on the Absenteeism of Health Workers from a Brazilian University Hospital. Health, 10, 853-877.

https://doi.org/10.4236/health.2018.106064

Received: January 28, 2018

Accepted: June 25, 2018

Published: June 28, 2018

Copyright ( 92018 by authors and Scientific Research Publishing Inc. This work is licensed under the Creative Commons Attribution International License (CC BY 4.0).

http://creativecommons.org/licenses/by/4.0/

(c) (7) Open Access

\begin{abstract}
Objective: Analyzing the association between Metabolic Syndrome (MS) risk factors and work absenteeism due to illnesses among University Hospital employees in Salvador City, Bahia, Brazil. Methods: Cross-sectional study comprised 1173 Bahia Federal University Hospital employees: 57.3\% of them were federal employees (statutory officers) and $42.7 \%$ were civil servants (CLT employees). Participants were in the age group 41.5 years: $69.4 \%$ were female and $30.6 \%$ were male. Results: MS prevalence in employees reached $6.6 \%$; women accounted for $69.7 \%$ of the total. The percentage increases with age and has similar proportion among direct-care providers and ancillary supports personnel, $40.8 \%$ and $39.5 \%$, respectively. The risk factor analysis showed $25 \%$ hypertension, $54.6 \%$ weight excess (18.4\% obese and $36.2 \%$ overweight patients), 20\% hypercholesterolemia, 9.9\% hypertriglyceridemia and $6.5 \%$ diabetes mellitus prevalence. Absenteeism longer than one day within the studied population reached $40.1 \%$. Osteoarticular diseases were the most common reason for absenteeism, accounting for $50.7 \%$ of it: $62.6 \%$ of whom were either overweight or obese. The mean cost with absenteeism per employee was $\mathrm{R} \$ 5492.87$ ( $\$ 2347.00$ US Dollars), and it corresponds to annual cost of $\mathrm{R}$ 2,587,139.53 ( $\$ 1,105,610.00$ US Dollars). The highest cost with absenteeism caused by illnesses was recorded for female workers; for federal employees who were patient caregivers, who worked in the Pediatric Centre and who worked at night shifts. Conclusion: The high Metabolic Syndrome risk factors and overweight incidence are responsible for the increased costs faced by hospitals due to high absenteeism rates.
\end{abstract}

\section{Keywords}

Metabolic Syndrome, Risk Factors, Absenteeism, University Hospital 


\section{Introduction}

The increased prevalence of Metabolic Syndrome (MS) risk factors and, consequently, of cardiovascular diseases (CVD) in health workers reached alarming global health and socioeconomic concern [1]-[7].

Several studies have shown that both MS and its risk factors (Obesity, Hypertension-HTN, Diabetes Mellitus-DM and Dyslipidemia) in health workers are responsible for increased absenteeism rates caused by illnesses, as well as for increased costs due to extra temporary personnel contracted, life expectance and quality reduction, productivity decrease and for patient care giving-quality impairment [1] [2] [6] [8] [9] [10].

The problem emerges from individual genetic factors and from environmental, political and management flaws in health institutions. Health workers are exposed to life and death duality in their work shifts, as well as to inappropriate working conditions, to work overload and stress; moreover, they are subjected to extra working shifts, to physical wear, to occupational risks and to other adverse conditions that favor the development of different diseases [11]-[16].

Absenteeism is recorded when the employee skips work due to unjustified particular reasons, to overall illness or to illness caused by professional reasons (legal and compulsory) [14]-[20].

Absenteeism prevalence among nurses, regardless of the cause [15] [16] [21] is well described in the literature. The absence of nurses generates a tremendous impact on the quality of the care provided to patients due to their expressive number in health institutions and, mainly, due to the direct care they give to them. Therefore, when it comes to systematic data about the absenteeism behavior of health professionals with expertise on other health fields, there are very few studies presenting systematic data measuring the behavior of workers in relation to their absenteeism [22].

It is worth emphasizing that absenteeism in public organizations [22], mainly in public universities is real; it is a major concern to direction boards because, besides generating increased public-resource losses, it endangers teaching and research activities.

Accordingly, knowing the prevalent causes of and seeking alternative solutions to reduce absenteeism resulting from illness conditions are crucial for managers and for those in charge of hospitals. Such causes and solutions have straight relation to care providers, to the satisfaction of human resources, to cost reduction, to increased productivity and to improvement in the quality and safety of the care given to patients.

\section{Materials and Methods}

\subsection{Study Design and Population}

The present cross-sectional study, was conducted in the University Hospital Complex (UH) of Bahia Federal University, Brazil. It is a large public hospital funded by the public health system (SUS) and it is focused on teaching, on re- 
search and on patient caregiving activities; it covers all medical specialties and subspecialties.

The initial sample comprised 2139 workers, who were invited to participate in the survey. The voluntary sample comprised 1173 (54.8\%) workers. The exclusion criteria were: not to have a formal employment contract, to be pregnant, to be away from work for medical or other license reason during the study period, and not to agree on participating in the survey. The excluded group corresponded to $45.2 \%$ of the initial sample (966 subjects).

\subsection{Data Collection}

Data used in the present study were provided by a study entitled "The prevalence of overweight and obesity among workers of the Hospital Universitário Professor Edgard Santos". Data were collected from October 2008 to October 2009.

The Health Clinic office was firstly implemented in order to have physical structure and to be equipped to develop health professionals' guidance and monitoring programs and to support research protocols focused on identifying cardiovascular and osteoarticular disease risks.

The direction board joined forces with the Human Resources Department and with UH/UFBA Communication Assessors to define strategies aiming at mobilizing workers to participate in this research project. Educational lectures on obesity, hypertension and healthy eating habits were given to the internal personnel; posters depicting the topics were elaborated and distributed in all $\mathrm{UH}$ sectors, as well as outspread through institutional media; all service centers were visited and direct contact by phone was made with many employees.

The research was approved by the Research Ethics Committee of HUPES Complex (Decree n. 99/2008). The free and informed consent form (ICF) was signed by the professionals participating in the research.

Data were collected by students and health professionals involved in the study after they received a previous full, and detailed, explanation about the nature, rationale, objective, methods and benefits of the study. The right to be informed about the partial and final results of the research was assured to the participants.

A questionnaire was applied in order to collect occupational and personal data (age, profession, work shift, working hours, licenses, medical certificates and work absence); as well as family member (family history of cardiovascular disease and comorbidities); and health-related data (physical activity, smoking, cardiovascular disease). Blood pressure-BP (digital and analogical), capillary glycaemia and anthropometric measurements (weight and height) were evaluated (Annex C). All participants had access to their examination results and received a booklet, and guidelines, about healthy eating from nutrition students and/or dietitians.

The employee was immediately referred to Emergency Room in case any significant clinical BP and/or blood glucose data were recorded during data collec- 
tion. Workers with overweight, obesity, hypertension or hyperglycemia diagnosis were referred to specific treatment centers located in the outpatient clinics.

Blood pressure was measured by nursing students, who were under the supervision of a professional nurse. The worker would sit on a chair and his/her non-dominant arm would lay on a hard surface, and his/her BP was measured after 5 to 10 minutes rest. Measurements were carried out with the aid of an analogic sphygmomanometer ( $2 \mathrm{mmHg}$ precision) and with a digital apparatus Microlife ${ }^{\oplus}$-model BP3BTO-a. Two measures were taken with both equipment and the mean values were recorded.

Anthropometric data were collected by two nutrition students, who were under the supervision of a professional dietitian. Body weight (kilogram) was measured on the portable digital scale WISO ${ }^{\circledR}$ model W801 (180 kg capacity, 100 $\mathrm{g}$ accuracy) which was properly calibrated. The subject would stand barefoot on the scale wearing his/her underwear, only. The subject would stand barefoot on the portable stadiometer Aturexata ${ }^{\oplus}$ (maximum height $213 \mathrm{~cm}$, metal rod, and $0.5 \mathrm{~cm}$ accuracy) to measure stature.

Body mass index $\left(\mathrm{BMI}=\right.$ weight $/$ Height $\left.^{2}\right)$ was calculated to assess the patients' nutritional status. According to WHO [23] criteria, workers presenting BMI between 25 to $29.9 \mathrm{Kg} / \mathrm{m}^{2}$ were overweight and those showing BMI $\geq 30 \mathrm{Kg} / \mathrm{m}^{2}$ were obese. Grade I ( 30 to $\left.34.9 \mathrm{Kg} / \mathrm{m}^{2}\right)$, II ( 35 to $\left.39.9 \mathrm{Kg} / \mathrm{m}^{2}\right)$ and III $(\geq 40$ $\mathrm{Kg} / \mathrm{m}^{2}$ ) obesity participants were gathered in a single group in order to make it more representative.

The MS employees were those who presented at least three risk factors [24]: Obesity (BMI $\left.\geq 30 \mathrm{~kg} / \mathrm{m}^{2}\right),(\mathrm{PA}) \geq 130 \mathrm{mmHg}$, and/or $85 \mathrm{mmHg}$; those who have self-reporting hypertension (taking medication), glucose intolerance, fasting glucose $\geq 100 \mathrm{mg} / \mathrm{dl}$; or those having self-reporting hyperglycemia (taking medication) or blood glucose $\geq 200 \mathrm{mg} / \mathrm{ml}$ in random determinations, and self-reporting hypertriglyceridemia and/or hypercholesterolemia.

With regard to the present study, absenteeism caused by illness meant the employee's absence from labor activities. It could be justified by documented medical leave, or not; pregnancy related licenses, and licenses related to elective or esthetic surgeries, were excluded.

Information about the number of days out from work due to a specific illness and confirmed by the Human Resources Service of HU/UFBA, regardless of initial self-report by the employee, was the purpose of the present study.

Among the causes of absenteeism-licenses and medical evaluations, some of them were categorized just as "self-reported" by workers. The self-reported causes of absenteeism were those where the absence reason was not confirmed by the Personnel Department and not having been provided, as requested, by their workers Medical Services.

The mean salary of each category indifferent work contracts and working hours was used to calculate the direct cost of absenteeism. It was made to estimate the daily cost imposed per professional category. Finally, daily wage values were multiplied by the number of days out of work of each professional category. 


\subsection{Definition of Variables}

It was necessary categorizing some variables for statistical analysis purpose:

Work unit-Employees are distributed in three units composing the Hospital Complex. They were categorized as HUPES (General Hospital) and CPPHO/AMN (Pediatric Center and Outpatient Unit).

Work sector-Employees were grouped in three sector types: direct care providers, management and support.

Work shift-Categorized as dayshift and nightshift.

Employment-HU/UFBA workers have multiple employment contract categories, they can be public employees hired under a single government regime (Statutory) and typical private sector hiring employees ruled by consolidated labor laws (Civil servant).

Professional category-HU/UFBA workers are distributed into many occupational categories, such as: Direct care providers-professionals in charge of patient caregiving activities; Management-professionals in managerial positions and functions; and Support-all the categories in charge of management, teaching, research and patient caregiving support activities such as maintenance, environmental services, transportation, etc.

Number of days out due to sick leave-1 day out of work was enough to record absenteeism due to illness.

Reasons related to sick leave, to licenses and to medical self-reported certificates were analyzed according to the major groups described in the International Classification of Diseases (ICD 10) [25].

\subsection{Statistical Analysis}

Absolute and relative frequencies were the categorical variables; mean and standard deviation were used whenever applicable. Person's Chi-square test was adopted to check the potential association between the variables of interest concerning the general characteristics of the studied population (gender, category and employment contract, activity sector, location and work shift) and the number of days under sick leave (absenteeism).

The Student's t-test was applied to compare the mean number of days out of work due to license (absenteeism) between categorical variable strata; the analysis of variance (ANOVA) was conducted when the strata exceeded two categories.

Prevalence ratio and 95\% confidence intervals were used for statistical inference and calculated by the Mantel-Haenszel method. The crude and stratified analyses were associated to measure the relation among the number of days out of work (absenteeism), metabolic syndrome risk factors and the metabolic syndrome itself.

Absenteeism prevalence in subjects with metabolic syndrome and in those who did not present it was calculated to estimate prevalence ratio, (PR) by the ratio between the two measurements. The association and the strength of the 
association between events were also investigated and evaluated at $95 \%$ confidence intervals (CI 95\%).

The Factorial Analysis of Multiple Correspondences (FAMC) is a statistical technique applied to studies of exploratory or descriptive nature and to inferential statistics (hypothesis testing). It is suitable to study a set of categorical variables, because this method allows to graphically analyzing the simultaneous relations between different categories in this set of variables. It is possible to visually assess the associations between qualitative variable extracts in FAMC graphics, which show the proximity between points (a strict analysis comprises a characteristic profile). Each point in the graphic represents the strata of variables assessed in the study.

The analysis consists of building a cloud of points within a multidimensional space designed for a factorial plan (Cartesian plane) in order to retain as much true data variability as possible-the real distance between the strata of the herein assessed variables. The first factorial plan is always the one that best preserves the real distance between the strata of the descriptive variable set. The closeness between two or more points belonging to different variable categories can be translated through the strong association between these categories [26].

Accordingly, this technique aims at verifying the professional category, activity sector, employment contract, and work unit and shift of hospital complex employees, as well as the association of their sex with metabolic syndrome and with its risk factors. It is worth mentioning that the main commitment to the application of this technique lies on relating all these variables to absenteeism. Therefore, the aim of the present study was to outline the FAMC profile of employees who have to work.

The $\mathrm{p}$ value $<0.05$ was considered statistically significant. Graphics were generated in Microsoft Excel (version 2007). The statistical analyses were conducted in the Statistical Package for Social Sciences (SPSS) 18.0 for Windows software.

\section{Results}

University Hospital Complex Professor Edgard Santos (HU/UFBA) employees' characteristics and their overall MS risk factor and absenteeism results between 2008 and 2009 are shown in Tables 1-3.

The sample comprised 1173 (54.8\%) HU/UFBA employees, of whom 814 (69.4\%) were women and 359 (30.6\%) were men in the age group 41.5 years (SD $=11.1)$ - minimum age 19 years and maximum age 69 years.

Ninety-nine occupational category types distributed in two training levels composed the current study (Table 1). Given the diversity of positions for analysis, the participants were grouped in three categories: direct care providers, management and support professionals.

Direct care provider professionals included 357 nursing technicians/assistants (30.4\%), 86 nurses (7.3\%), 24 dietitians (2.0\%), 22 physical therapists (1.9\%), 20 physicians (1.7\%), 17 social workers (1.4\%), 20 pharmacists (1.7\%), 03 biologists (0.3\%), 39 pharmacy assistants (3.3\%), 39 auxiliary/lab technicians (3.3\%), 
Table 1. Characteristics of the HU/UFBA employee sample by professional category and training level between 2008 and 2009, Salvador-Bahia.

\begin{tabular}{cccccccc}
\hline \multirow{2}{*}{ Professional Category } & \multicolumn{4}{c}{ Training Level } & \multicolumn{2}{c}{ Total } \\
\cline { 2 - 6 } & \multicolumn{2}{c}{ Higher Education } & \multicolumn{2}{c}{ High School } & \multicolumn{2}{c}{} \\
\cline { 2 - 6 } & $\mathrm{N}=\mathbf{2 4 6}$ & $21.0 \%$ & $\mathrm{~N}=\mathbf{9 2 7}$ & $\mathbf{7 9 . 0 \%}$ & $\mathrm{N}=1.173$ & $\%$ \\
\hline Management & 43 & 17,5 & 241 & 26.0 & 284 & 24.2 \\
Direct care providers & 203 & 82.5 & 423 & 45.6 & 626 & 53.4 \\
Support & 0 & --- & 263 & 28.4 & 263 & 22.4 \\
\hline
\end{tabular}

$\mathrm{n}=$ Number of observations.

among others. When it comes to management professionals, 173 administrative assistants (14.7\%), 3.0 accountants (0.3\%), 17 archivists (1.4\%), 13 receptionists (1.1\%), 8 cashiers $(0.7 \%), 05$ computer analysts/programmers $(0.5 \%)$, and personnel management analysts $(0.3 \%)$ participated in the research. Forty-seven (47) environmental service assistants-AOSD (4.0\%), 46 kitchen assistants (3.9\%), 27 laundry assistants (2.3\%), 15 security officers (1.3\%), 12 drivers (1.0\%), 10 doormen $(0.9 \%), 8.0$ Cookers (0.7\%), among others, participated in the study.

The participants are enrolled in 137 different services and sectors such as inpatient Units, ICU, Outpatient, environmental services, laundry, maintenance, pharmacy, medical services, social work, medical records, laboratories, diagnostic services, among others. They were grouped by activity sector as management, direct care provider and support for analysis purposes.

The total of diseases self-reported by workers was 233 (20.3\%) HTN, 225 (20\%) Hypercholesterolemia, 110 (9.9\%) Hypertriglyceridemia, 56 (4.8\%) DM, and 38 (3.2\%) ACD cases. Family history of HTN, DM, ACD and obesity represented 863 (75.3\%), 542 (47.3\%), 457 (39.8\%) and 352 (30.7\%) of the cases, respectively.

The prevalent MS risk factors in the studied population were hypertension 284 (25.0\%), hypercholesterolemia 225 (20.0\%), obesity 214 (18.4\%), hypertriglyceridemia 110 (9.9\%) and DM 73 (6.5\%) (Table 2).

The HTN rate was similar in both sexes, and in the direct care providers and support categories. There was association between risk factor and its variables ( $\mathrm{p}$-value $=0.000)$. Hypertension was more prevalent among support workers 103 (39.9\%), assistant/technician nurses 63 (22.2\%) and management assistants 41.0 (14.4\%).

Overweight was observed in 634 (54\%) workers, who were distributed in 214 $(18.4 \%)$ obese (z-score $\geq 3$ ) and $420(35.6 \%)$ overweight (z-score $\geq 1$ and $<2$ ). There was statistically significant association between obesity and nightshift ( $\mathrm{p}$-value $=0.000)$. Interestingly, $47(74.6 \%)$ direct care providers in the nightshift were overweight.

Obesity had similar prevalence among management and support workers, and this number corresponded to $50(23.5 \%)$ and $46(21.6 \%)$ of the workers, respectively. It was more prevalent among direct care provider workers, 117 (54.9\%). 
Table 2. Characteristics of the HU/UFBA employees sample and its overall MS risk factors results between 2008 and2009, Salvador-Bahia.

\begin{tabular}{|c|c|c|c|c|c|c|c|c|c|c|c|c|c|c|c|c|}
\hline \multirow{2}{*}{ Variable } & \multicolumn{2}{|c|}{$\begin{array}{l}\text { Metabolic } \\
\text { Syndrome }\end{array}$} & \multicolumn{2}{|c|}{ HTN } & \multicolumn{2}{|c|}{$\mathrm{DM}$} & \multicolumn{2}{|c|}{ Hypertriglyceridemia } & \multicolumn{2}{|c|}{ Hypercholesterolemia } & \multicolumn{2}{|c|}{ Obesity } & \multicolumn{2}{|c|}{ Sedentary } & \multicolumn{2}{|c|}{ Smoking } \\
\hline & $\mathbf{n}$ & $\%$ & $\mathrm{n}$ & $\%$ & $\mathrm{n}$ & $\%$ & $\mathrm{~N}$ & $\%$ & $\mathbf{N}$ & $\%$ & $\mathbf{n}$ & $\%$ & $\mathbf{n}$ & $\%$ & $\mathrm{n}$ & $\%$ \\
\hline General & 76 & 6.6 & 284 & 25.0 & 73 & 6.5 & 110 & 9.9 & 225 & 20.0 & 214 & 18.4 & 756 & 64.6 & 59 & 5. 1 \\
\hline \multicolumn{17}{|l|}{ Sex } \\
\hline Male & 23 & 30.3 & 133 & 46.8 & 25 & 34.2 & 33 & 30.0 & 53 & 23.6 & 43 & 20.2 & 187 & 24.7 & 20 & 33.9 \\
\hline \multicolumn{17}{|l|}{$\begin{array}{c}\text { Professional } \\
\text { category }\end{array}$} \\
\hline $\begin{array}{c}\text { Patient care } \\
\text { provider }\end{array}$ & 31 & 40.8 & 111 & 39.1 & 32 & 43.8 & 59 & 53.6 & 125 & 55.6 & 117 & 54.9 & 410 & 54.2 & 24 & 40.7 \\
\hline Support & 30 & 39.5 & 103 & 36.3 & 29 & 39.7 & 26 & 23.6 & 54 & 24.0 & 50 & 23.5 & 162 & 21.4 & 21 & 35.6 \\
\hline \multicolumn{17}{|l|}{ Work Sector } \\
\hline $\begin{array}{c}\text { Patient } \\
\text { caregiving }\end{array}$ & 44 & 57.9 & 139 & 48.9 & 45 & 61.6 & 66 & 60.0 & 156 & 69.3 & 144 & 67.6 & 496 & 65.6 & 32 & 54.2 \\
\hline Management & 7 & 9.2 & 44 & 15.5 & 6 & 8.2 & 12 & 10.9 & 22 & 9.8 & 23 & 10.8 & 80 & 10.6 & 8 & 13.6 \\
\hline Support & 25 & 32.9 & 101 & 35.6 & 22 & 30.1 & 32 & 29.1 & 47 & 20.9 & 46 & 21.6 & 180 & 23.8 & 19 & 32.2 \\
\hline \multicolumn{17}{|l|}{$\begin{array}{c}\text { Employment } \\
\text { contract }\end{array}$} \\
\hline Statutory ${ }^{\star}$ & 52 & 68.4 & 164 & 57.7 & 58 & 79.5 & 77 & 70.0 & 152 & 67.6 & 134 & 62.9 & 427 & 56.5 & 37 & 62.7 \\
\hline Civil Servant ${ }^{* *}$ & 24 & 3.6 & 120 & 42.3 & 15 & 20.5 & 33 & 30.0 & 73 & 32.4 & 79 & 37.1 & 329 & 43.5 & 22 & 37.3 \\
\hline CPPHO/AMN & 25 & 32.9 & 85 & 29.9 & 28 & 38.4 & 40 & 36.4 & 78 & 34.7 & 71 & 33.3 & 225 & 29.8 & 16 & 27.1 \\
\hline \multicolumn{17}{|l|}{ Work Shifts } \\
\hline Day & 68 & 89.5 & 256 & 90.1 & 65 & 89.0 & 100 & 90.9 & 200 & 88.9 & 178 & 83.6 & 681 & 90.1 & 53 & 89.8 \\
\hline Night & 8 & 10.5 & 28 & 9.9 & 8 & 11.0 & 10 & 9.1 & 25 & 11.1 & 35 & 16.4 & 75 & 9.9 & 6 & 10.2 \\
\hline
\end{tabular}

HU/UFBA: Complexo hospitalar universitário professor edgard santos. HTN: Hypertension. DM: Diabetes mellitus. n: n. of individuals. ${ }^{\star}$ Statutory—public employees hired under a single legal regime and ${ }^{\star *}$ Civil Servants—typical private sector employee ruled by consolidated Brazilian labor laws.

However, the highest obesity prevalence was observed in assistant nursing technicians, 87 (40.8\%); in management assistants, 24 (11.3\%); and in kitchen assistants, $12(5.6 \%)$.

High Dyslipidemia rates were recorded between nursing technicians, 109 (63.3\%); and management assistants, 43 (26.0\%). Only five physicians presented hypertriglyceridemia (5.5\%).

The overall prevalence of Metabolic Syndrome in HU/UFBA employees was 76 (6.6\%); it was much higher in women, 53 (69.7\%), than in men, $23(30.3 \%)$; however, it was similar between the patient caregiving, 31 (40.8\%); and support, $30(39.5 \%)$ employees (Table 2). With regard to nursing technicians and management assistants, $26(34.2 \%)$ and $9(11.8 \%)$ presented MS, respectively. 
The global absenteeism prevalence was $40.1 \%$ (471), it was higher in direct care providers, $306(65 \%)$; and similar between management and support employees, 86 (18.2) and 79 (16.8\%), respectively (Table 3).

Similarly, when the number of days out of work was equal to or larger than 19 days MS prevalence was higher indirect care providers, 83 (76.1\%).

Nurses, nursing assistants/technicians, kitchen assistants, laundry assistants and management assistants presented $\geq 1$ day sick leave; 54 (62.8\%), 202 (56.6\%); 21 (45.7\%); 10 (37\%) and 53 (30.6\%), respectively.

Absenteeism prevalence was higher in extra-shift employees, $\geq 30 \mathrm{~h} 102$ (53\%) than in those following regular work shifts, 29 (35.4\%).

Among the individuals in the age group 41 years or less (574, in total), 271 (47.2\%) recorded absenteeism prevalence $\geq 1$ day, and those in the age 42 years or older (200, in total) recorded $33.4 \%$ MS prevalence $(\mathrm{P}$-value $=0.0001)$.

Gender, professional category and work sector were associated with absenteeism $(\mathrm{p}$-value $=0.0001)($ Table 3$)$.

Table 3. General characteristics of the studied population according to absenteeism $(\mathrm{n}=$ 1173).

\begin{tabular}{|c|c|c|c|c|c|c|}
\hline \multirow{3}{*}{ Variable } & \multicolumn{4}{|c|}{ Absenteeism } & \multirow{2}{*}{\multicolumn{2}{|c|}{ Total }} \\
\hline & \multicolumn{2}{|c|}{ Yes } & \multicolumn{2}{|c|}{ No } & & \\
\hline & $\mathrm{N}=471$ & $40.1 \%$ & $\mathrm{~N}=702$ & $59.9 \%$ & $\mathrm{~N}=1173$ & $\%$ \\
\hline \multicolumn{7}{|l|}{$\operatorname{Sex}^{*}$} \\
\hline Female & 388 & 82.4 & 426 & 60.7 & 814 & 69.4 \\
\hline Male & 83 & 17.6 & 276 & 40.3 & 359 & 30.6 \\
\hline \multicolumn{7}{|l|}{ Professional Category* } \\
\hline Patient care provider & 306 & 65.0 & 320 & 45.6 & 626 & 53.4 \\
\hline Management & 86 & 18.2 & 198 & 28.2 & 284 & 24.2 \\
\hline Support & 79 & 16.8 & 184 & 26.2 & 263 & 22.4 \\
\hline \multicolumn{7}{|l|}{ Work Sector ${ }^{*}$} \\
\hline Patient care provider & 365 & 77.5 & 388 & 55.3 & 753 & 64.2 \\
\hline Management & 25 & 5.3 & 105 & 14.9 & 130 & 11.1 \\
\hline Support & 81 & 17.2 & 209 & 29.8 & 290 & 24.7 \\
\hline \multicolumn{7}{|l|}{ Employment contract** } \\
\hline Statutory & 267 & 56.7 & 405 & 57.7 & 672 & 57.3 \\
\hline Civil servant & 204 & 43.3 & 297 & 42.3 & 501 & 42.7 \\
\hline \multicolumn{7}{|l|}{ Unit*** } \\
\hline HUPES & 321 & 68.2 & 474 & 67.5 & 795 & 67.8 \\
\hline CPPHO/AMN & 150 & 31.8 & 228 & 32.5 & 378 & 32.2 \\
\hline \multicolumn{7}{|l|}{ Workshifts**** } \\
\hline Day & 423 & 89.8 & 648 & 92.3 & 1.071 & 91.3 \\
\hline Night & 48 & 10.2 & 54 & 7.7 & 102 & 8.7 \\
\hline
\end{tabular}

$\mathrm{n}=$ Number of observations. Person's Chi-Square $\mathrm{p}$-value. ${ }^{*} \mathrm{p}=0.0001,{ }^{* *} \mathrm{p}=0.7332,{ }^{* * *} \mathrm{p}=0.8205,{ }^{* * *} \mathrm{p}=$ 0.1365 . 
When the number of days out of work was related to the employment contract, prevalence of long-term license (19 days or more) was much higher in "statutory" employees, whereas short-term license (1 to 3 days) prevailed in "civil servant" employees (Figure 1).

Special attention should be given to outliers in both groups when results are interpreted. The "statutory" data series showed larger number of outliers, few workers stayed out of work for more than 200 days. Of the "civil servant" group recorded extreme values related to two individuals, who stayed 200 days out of work.

Results seem to confirm that longer absence was recorded for "statutory" employees. Results presented in Table 4 indicate greater absenteeism prevalence in employees with obesity (PR: 1.38, CI: -1.81 1.05) and hypercholesterolemia (PR: 1.56, CI: -2.29 1.06). HTN (PR: 0.78, CI: 0.66 - 0.94) was not determinant of high absenteeism. No statistically significant association was observed between the prevalence of the other herein assessed variables.

Sedentary employees had gross association with absenteeism (PR: 1.18, CI: -1.38 1.01), and it was more prevalent in "statutory" (PR: 1.24, CI: 1.01 - 1.53) employees working in patient care giving (PR: 1.20, CI: $-1.421 .02)$ at dayshift (PR: 1.20, CI: -1.41 1.02) in HUPES-General Hospital-(PR: 1.28, CI: -1.55 1.05). Smokers were more prevalent at nightshift (PR: 1.83, CI: -2.78 1.20) (Table 5).

Civil servants had higher absenteeism prevalence in the younger population (21 - 46 years). The most frequent periods of absence and occupational categories were the short ones, between 1 and 3 days out of work, and it corresponded

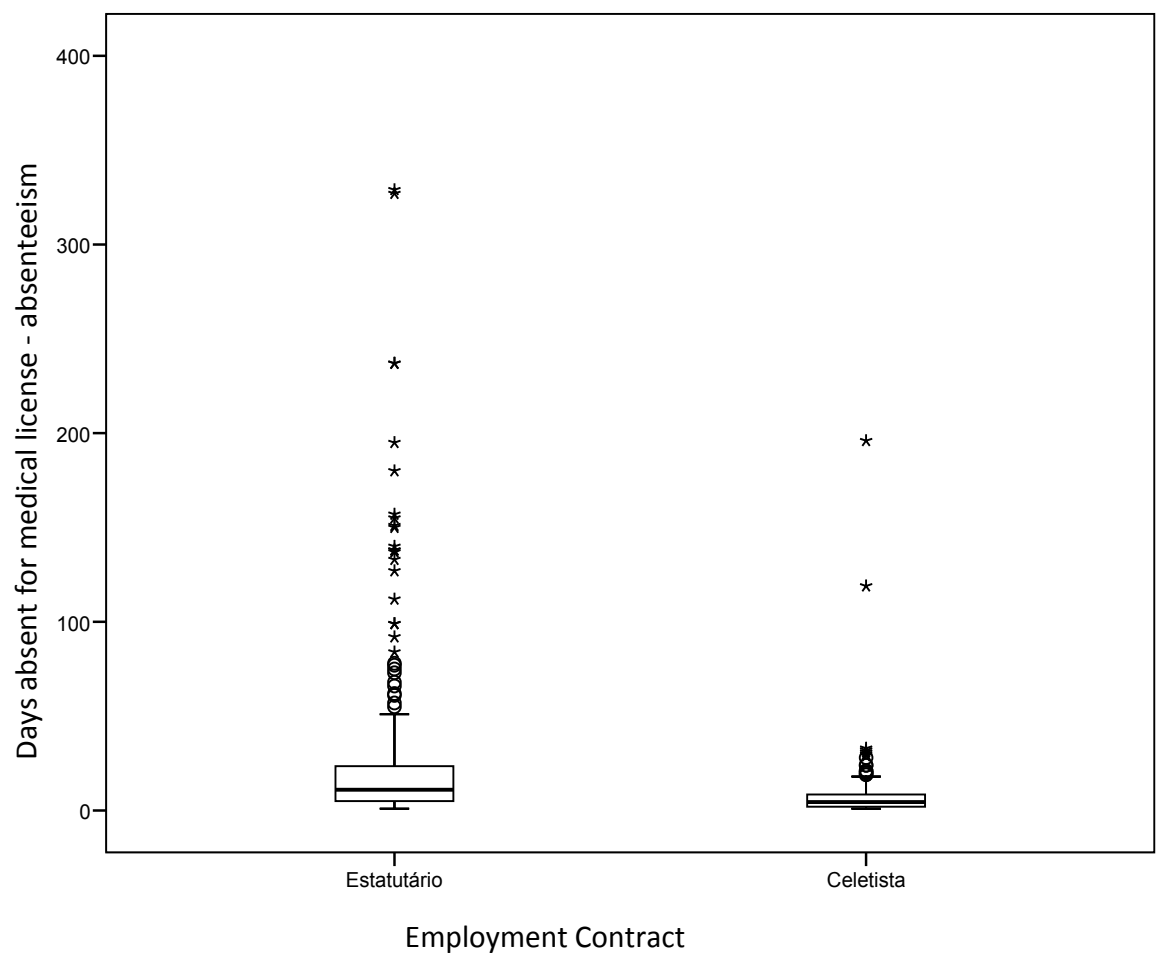

Figure 1. Days out of work according to the employees' employment contract. 
Table 4. Absenteeism prevalence, prevalence ratio at 95\% confidence intervals of the association between MS risk factors and absenteeism.

\begin{tabular}{|c|c|c|c|c|c|c|c|}
\hline \multirow{2}{*}{ Variable } & \multirow{2}{*}{ Prevalence } & \multirow{2}{*}{$\begin{array}{c}\begin{array}{c}\text { Metabolic } \\
\text { Syndrome }\end{array} \\
\text { RP }(95 \% \mathrm{CI})\end{array}$} & \multirow{2}{*}{$\begin{array}{c}\text { HTN } \\
\text { RP }(95 \% \mathrm{CI})\end{array}$} & \multirow{2}{*}{$\begin{array}{c}\mathrm{DM} \\
\mathrm{RP}(95 \% \mathrm{CI})\end{array}$} & \multirow{2}{*}{$\begin{array}{c}\text { Hypertriglyceridemia } \\
\text { RP }(95 \% \mathrm{CI})\end{array}$} & \multirow{2}{*}{$\begin{array}{c}\text { Hypercholesterolemia } \\
\text { RP ( } 95 \% \text { CI) }\end{array}$} & \multirow{2}{*}{$\begin{array}{c}\text { Obesity } \\
\text { RP }(95 \% \mathrm{CI})\end{array}$} \\
\hline & & & & & & & \\
\hline Gross Association & 40.1 & $\begin{array}{c}0.94 \\
(0.70-1.27)\end{array}$ & $\begin{array}{c}0.78 \\
(0.66-0.94)\end{array}$ & $\begin{array}{c}0.94 \\
(0.70-1.27)\end{array}$ & $\begin{array}{c}0.86 \\
(0.66-1.12)\end{array}$ & $\begin{array}{c}1.03 \\
(0.87-1.23)\end{array}$ & $\begin{array}{c}1.14 \\
(0.96-1.35)\end{array}$ \\
\hline \multicolumn{8}{|l|}{ Sex } \\
\hline Female & 47.7 & $\begin{array}{c}0.94 \\
(0.69-1.28)\end{array}$ & $\begin{array}{c}0.95 \\
(0.78-1.14)\end{array}$ & $\begin{array}{c}0.95 \\
(0.69-1.30)\end{array}$ & $\begin{array}{c}0.88 \\
(0.67-1.16)\end{array}$ & $\begin{array}{c}0.98 \\
(0.82-1.17)\end{array}$ & $\begin{array}{c}1.08 \\
(0.91-1.28)\end{array}$ \\
\hline Male & 23.1 & $\begin{array}{c}0.94 \\
(0.42-2.09)\end{array}$ & $\begin{array}{c}0.75 \\
(0.50-1.14)\end{array}$ & $\begin{array}{c}1.02 \\
(0.49-2.10)\end{array}$ & $\begin{array}{c}0.76 \\
(0.36-1.60)\end{array}$ & $\begin{array}{c}1.03 \\
(0.61-1.72)\end{array}$ & $\begin{array}{c}0.90 \\
(0.49-1.66)\end{array}$ \\
\hline \multicolumn{8}{|l|}{$\begin{array}{c}\text { Professional } \\
\text { Category }\end{array}$} \\
\hline $\begin{array}{l}\text { Patient care } \\
\text { provider }\end{array}$ & 48.9 & $\begin{array}{c}0.99 \\
(0.68-1.43)\end{array}$ & $\begin{array}{c}0.93 \\
(0.75-1.16)\end{array}$ & $\begin{array}{c}0.95 \\
(0.65-1.39)\end{array}$ & $\begin{array}{c}1.06 \\
(0.58-1.94)\end{array}$ & $\begin{array}{c}0.93 \\
(0.75-1.15)\end{array}$ & $\begin{array}{c}1.11 \\
(0.91-1.34)\end{array}$ \\
\hline Management & 30.3 & $\begin{array}{c}0.42 \\
(0.11-1.54)\end{array}$ & $\begin{array}{c}0.62 \\
(0.38-1.01)\end{array}$ & $\begin{array}{c}1.06 \\
(0.47-2.41)\end{array}$ & $\begin{array}{c}0.72 \\
(0.50-1.02)\end{array}$ & $\begin{array}{c}0.88 \\
(0.53-1.45)\end{array}$ & $\begin{array}{c}0.93 \\
(0.56-1 . .3)\end{array}$ \\
\hline Support & 30.0 & $\begin{array}{c}1.40 \\
(0.86-2.27)\end{array}$ & $\begin{array}{c}0.89 \\
(0.61-1.31)\end{array}$ & $\begin{array}{c}1.03 \\
(0.58-1.84)\end{array}$ & $\begin{array}{c}1.23 \\
(0.70-2.18)\end{array}$ & $1.56(1.06-2.29)$ & $\begin{array}{c}1.43 \\
(0.96-2.14)\end{array}$ \\
\hline \multicolumn{8}{|l|}{ Work Sector } \\
\hline $\begin{array}{l}\text { Patient care } \\
\text { provider }\end{array}$ & 48.5 & $\begin{array}{c}1.08 \\
(0.81-1.45)\end{array}$ & $\begin{array}{c}0.97 \\
(0.80-1.18)\end{array}$ & $\begin{array}{c}1.05 \\
(0.78-1.41)\end{array}$ & $\begin{array}{c}0.85 \\
(0.63-1.15)\end{array}$ & $\begin{array}{c}1.02 \\
(0.85-1.23)\end{array}$ & $\begin{array}{c}1.13 \\
(0.95-1.35)\end{array}$ \\
\hline Management & 19.2 & --- & $\begin{array}{c}0.64 \\
(0.27-1.51)\end{array}$ & --- & $\begin{array}{c}0.40 \\
(0.06-2.70)\end{array}$ & $\begin{array}{c}0.40 \\
(0.10-1.57)\end{array}$ & $\begin{array}{c}1.15 \\
(0.48-2.75)\end{array}$ \\
\hline Support & 27.9 & $\begin{array}{c}0.84 \\
(0.41-1.72)\end{array}$ & $\begin{array}{c}0.71 \\
(0.47-1.08)\end{array}$ & $\begin{array}{c}0.77 \\
(0.35-1.71)\end{array}$ & $\begin{array}{c}1.12 \\
(0.64-1.95)\end{array}$ & $\begin{array}{c}1.12 \\
(0.70-1.79)\end{array}$ & $\begin{array}{c}1.01 \\
(0.61-1.66)\end{array}$ \\
\hline \multicolumn{8}{|l|}{$\begin{array}{c}\text { Employment } \\
\text { contract }\end{array}$} \\
\hline Statutory & 39.7 & $\begin{array}{c}0.76 \\
(0.50-1.15)\end{array}$ & $\begin{array}{c}0.83 \\
(0.65-1.04)\end{array}$ & $\begin{array}{c}0.90 \\
(0.63-1.28)\end{array}$ & $\begin{array}{c}0.87 \\
(0.63-1.20)\end{array}$ & $\begin{array}{c}1.01 \\
(0.81-1.26)\end{array}$ & $\begin{array}{c}1.20 \\
(0.97-1.48)\end{array}$ \\
\hline Civil Servant & 40.7 & $\begin{array}{c}1.35(0.92- \\
1,98)\end{array}$ & $\begin{array}{c}0.73 \\
(0.55-0.97) \\
\end{array}$ & $\begin{array}{c}1.13 \\
(0.65-1.96) \\
\end{array}$ & $\begin{array}{c}0.82 \\
(0.50-1.35) \\
\end{array}$ & $\begin{array}{c}1.08 \\
(0.81-1.43) \\
\end{array}$ & $\begin{array}{c}1.06 \\
(0.80-1.41)\end{array}$ \\
\hline \multicolumn{8}{|l|}{ Unit } \\
\hline HUPES & 40.4 & $\begin{array}{c}1.02 \\
(0.72-1.43)\end{array}$ & $\begin{array}{c}0.75 \\
(0.60-0.93)\end{array}$ & $\begin{array}{c}1.04 \\
(0.73-1.48)\end{array}$ & $\begin{array}{c}0.96 \\
(0.71-1.31)\end{array}$ & $\begin{array}{c}1.02 \\
(0.82-1.26)\end{array}$ & $\begin{array}{c}1.03 \\
(0.83-1.28)\end{array}$ \\
\hline СРPHO/AMN & 39.7 & $\begin{array}{c}0.79 \\
(0.44-1.42) \\
\end{array}$ & $\begin{array}{c}0.87 \\
(0.63-1.19) \\
\end{array}$ & $\begin{array}{c}0.78 \\
(0.45-1.36) \\
\end{array}$ & $\begin{array}{c}0.68 \\
(0.40-1.14) \\
\end{array}$ & $\begin{array}{c}1.06 \\
(0.78-1.43) \\
\end{array}$ & $\begin{array}{c}1.38 \\
(1.05-1.81)\end{array}$ \\
\hline \multicolumn{8}{|l|}{ Work shifts } \\
\hline Day & 39,5 & $\begin{array}{c}0.92 \\
(0.67-1.27)\end{array}$ & $\begin{array}{c}0.76 \\
(0.63-0.92)\end{array}$ & $\begin{array}{c}0.96 \\
(0.70-1.31)\end{array}$ & $\begin{array}{c}0.83 \\
(0.62-1.10)\end{array}$ & $\begin{array}{c}1.03 \\
(0.86-1.24)\end{array}$ & $\begin{array}{c}1.12 \\
(0.93-1.35)\end{array}$ \\
\hline Night & 47,1 & $\begin{array}{c}1.07 \\
(0.52-2.22)\end{array}$ & $\begin{array}{c}0.97 \\
(0.61-1.55)\end{array}$ & $\begin{array}{c}0.80 \\
(0.32-2.00)\end{array}$ & $\begin{array}{c}1.14 \\
(0.59-2.22)\end{array}$ & $\begin{array}{c}0.99 \\
(0.59-1.65)\end{array}$ & $\begin{array}{c}1.14 \\
(0.75-1.73)\end{array}$ \\
\hline
\end{tabular}

The $95 \%$ confidence intervals were calculated through the Mantel-Haenszel method.

to $83(40.7 \%)$. They differed from "statutory", who recorded more absences due to illness at the age group 34 to 69 years and larger number of days out of work (19 days or more), 74 (79.6\%). 
Table 5. Absenteeism prevalence, prevalence ratio at $95 \%$ confidence intervals of the association between physical inactivity and smoking, and absenteeism.

\begin{tabular}{|c|c|c|c|}
\hline \multirow{2}{*}{ Variable } & \multirow{2}{*}{ Prevalence } & \multirow{2}{*}{$\begin{array}{c}\text { Sedentary } \\
\text { RP }(95 \% \mathrm{CI})\end{array}$} & \multirow{2}{*}{$\begin{array}{c}\text { Smoking } \\
\text { RP }(95 \% \mathrm{CI})\end{array}$} \\
\hline & & & \\
\hline Gross Association & 40.1 & $1.18(1.01-1.38)$ & $1.10(0.82-1.48)$ \\
\hline \multicolumn{4}{|l|}{ Sex } \\
\hline Female & 47.7 & $1.12(0.95-1.32)$ & $1.13(0.84-1.53)$ \\
\hline Male & 23.1 & $0.90(0.62-1.31)$ & $1.08(0.49-2.36)$ \\
\hline \multicolumn{4}{|l|}{ Professional Category } \\
\hline Patient care provider & 48.9 & $1.16(0,98-1,39)$ & $1.28(0.93-1.77)$ \\
\hline Management & 30.3 & $1.37(0,91-2,05)$ & $1.19(0,57-2,46)$ \\
\hline Support & 30.0 & $1.02(0,70-1,49)$ & $0.95(0.47-1.92)$ \\
\hline \multicolumn{4}{|l|}{ Work Sector } \\
\hline Patient care provider & 48.5 & $1.20(1.02-1.42)$ & $1.30(0.98-1.72)$ \\
\hline Management & 19.2 & $1.61(0.72-3.57)$ & $0.65(0.10-4.23)$ \\
\hline Support & 27.9 & $0.89(0.61-1.29)$ & $0.93(0.43-2.02)$ \\
\hline \multicolumn{4}{|l|}{ Employment contract } \\
\hline Statutory & 39.7 & $1.24(1.01-1.53)$ & $1.02(0.68-1.52)$ \\
\hline Civil servant & 40.7 & $1.10(0.88-1.39)$ & $1.24(0.80-1.90)$ \\
\hline \multicolumn{4}{|l|}{ Unit } \\
\hline HUPES & 40.4 & $1.28(1,05-1,55)$ & $1.03(0.72-1.49)$ \\
\hline CPPHO/AMN & 39.7 & $1.02(0.79-1.32)$ & $1.27(0.76-2.11)$ \\
\hline \multicolumn{4}{|l|}{ Work shifts } \\
\hline Day & 39.5 & $1.20(1.02-1.41)$ & $1.00(0.71-1.41)$ \\
\hline Night & 47.1 & $0.97(0.61-1.54)$ & $1.83(1.20-2.78)$ \\
\hline
\end{tabular}

$\mathrm{PR}=$ Prevalence ratio. $\mathrm{CI}=$ Confidence intervals. The $95 \%$ confidence intervals were calculated through the Mantel-Haenszel method.

In total, 8.935 working days were lost during the 12 -month data collection period. The overall mean number of days out of work was $19.0(\mathrm{SD}=37.8): 27.1$ $(\mathrm{SD}=46.4)$ by "statutories" and $8.4(\mathrm{DP}=16.7)$ by "civil servants". Similar values were recorded for the General Hospital: 18.2 ( $\mathrm{SD}=38.8$ ); and CPPHO/AMN (Pediatric Center and Outpatient Unit): $20.5(\mathrm{SD}=35.5)$. And the number was much larger among nightshift workers 25.8 ( DP = 43.4\%) (Table 6). Cost data related to absenteeism are shown in Table 7.

When the reasons for sick leave absenteeism were analyzed by excluding the licenses and certificates associated with pregnancy, as well as the elective and esthetics surgeries, the second most prevalent cause of it lied on Osteoarticular diseases, 138 (19.5\%); which were followed by contagious and viral infectious diseases $(159 ; 22.5 \%)$.

Osteoarticular diseases caused absenteeism mainly among patient care providers 91 (65.9\%); in those who worked in dayshifts, 115 (83.3\%); "statutories", 105 (76.1\%); sedentary, 97 (70.3\%), General Hospital employees, 84 (60.9\%); and 
Table 6. Mean, standard deviation, median, 25 and 75 percentile, maximum and minimum values and total days out of work estimates $(n=471)$ between 2008 and 2009.

\begin{tabular}{|c|c|c|c|c|}
\hline Variable & Mean (SD) & $\begin{array}{c}\text { Median } \\
\text { (centiles 25 - 75) }\end{array}$ & $\begin{array}{l}\text { Minimal - } \\
\text { Maximo }\end{array}$ & Total \\
\hline Geral & $19.0(37.8)$ & $7.0(3.0-18.0)$ & $1-329$ & 8,935 \\
\hline \multicolumn{5}{|l|}{ Sex ${ }^{*}$} \\
\hline Female & $19.7(36.5)$ & $8.0(3.0-20.0)$ & $1-327$ & 7650 \\
\hline Male & $15.5(43.1)$ & $7.0(3.0-11.0)$ & $1-329$ & 1285 \\
\hline \multicolumn{5}{|l|}{ Professional Category ${ }^{* * 2,3}$} \\
\hline Patient care provider & $21.5(40.1)$ & $8.0(3.0-20.0)$ & $1-327$ & 6572 \\
\hline Management & $10.5(20.8)$ & $5.0(2.0-8.0)$ & $1-137$ & 900 \\
\hline Support & $18.5(41.7)$ & $8.0(4.0-15.0)$ & $1-329$ & 1463 \\
\hline \multicolumn{5}{|l|}{ Work Sector ${ }^{* * *}{ }^{2}$} \\
\hline Patient care provider & $19.4(36.7)$ & $8.0(3.0-19.0)$ & $1-327$ & 7093 \\
\hline Management & $7.3(6.2)$ & $7.0(3.0-8.0)$ & $1-31$ & 182 \\
\hline Support & $20.5(47.0)$ & $7.0(3.0-15.0)$ & $1-329$ & 1660 \\
\hline \multicolumn{5}{|l|}{ Employment contract $* * * * 1$} \\
\hline Statutory & $27.1(46.4)$ & $11.0(5.0-24.0)$ & $1-329$ & 7225 \\
\hline Civil Servant & $8.4(16.7)$ & $4.5(2.0-8.5)$ & $1-196$ & 1,710 \\
\hline \multicolumn{5}{|l|}{ Unit ${ }^{* * * * * 1}$} \\
\hline HUPES & $18.2(38.8)$ & $7.0(3.0-16.0)$ & $1-329$ & 5857 \\
\hline CPPHO/AMN & $20.5(35.5)$ & $8.0(3.0-20.0)$ & $1-237$ & 3078 \\
\hline \multicolumn{5}{|l|}{ Work shifts $* * * * * * 1$} \\
\hline Day & $18.2(37.1)$ & $7.0(3.0-17.0)$ & $1-329$ & 7698 \\
\hline Night & $25.8(43.4)$ & $7.5(3.5-26.0)$ & $1-196$ & 1237 \\
\hline
\end{tabular}

${ }^{1}$ Mann-Whitney Test. ${ }^{2}$ Kruskal-Wallis Test. ${ }^{3}$ According to Holms test, the professional categories' "Patient care provider" and "Management", as well as "Management" and "Support" were statistically different. ${ }^{*}(\mathrm{p}$ $=0.0313),{ }^{* *}(\mathrm{p}=0.0014),{ }^{* * *}(\mathrm{p}=0.2000),{ }^{* * * *}(\mathrm{p}-\mathrm{valor}=0.0001),{ }^{* * * * *}(\mathrm{p}-\mathrm{valor}=0.2361),{ }^{* * * * * *}(\mathrm{p}-$ valor $=$ $0.3734)$.

women, $113(81.8 \%)$. It is worth mentioning that gender, professional category and employment contract were statistically significant to the prevalence of Osteoarticular diseases $(\mathrm{p}$-value $=0.000)$.

Once again, the high prevalence of Osteoarticular disease in nursing technicians/assistants, 69 (50.0\%), call the attention; it was followed by management assistants, 12 (8.7\%); and by nurses, 8 (5.8\%)-the number are statistically significant ( $\mathrm{p}$-value $=0.0002$ ).

In addition to the reasons mentioned above, data evidenced that the reasons for absence due to sick leave justified by workers according to the $\mathrm{ICD}^{25}$ groups, were: symptoms, signs, abnormal findings, 120 (16.9\%); and other causes, 291 (41.1\%); among others.

Absences caused by social factors (sick relative monitoring, second job, travel, study, among others) corresponded to 110 (9.5\%), of whom 60 (54.3\%) were "statutory" and 50 (45.7\%) were "civil servants". 
Table 7. Mean individual and total cost per year $(n=471)$.

\begin{tabular}{|c|c|c|}
\hline Variable & Mean $(R \$)$ & Total (R\$) \\
\hline General & 5492.87 & $2,587,139.53$ \\
\hline \multicolumn{3}{|l|}{ Sex } \\
\hline Female & 6181.85 & $2,398,556.20$ \\
\hline Male & 2272.09 & $188,583.33$ \\
\hline \multicolumn{3}{|l|}{ Professional Category } \\
\hline Patient care provider & 7750.81 & $2,371,747.24$ \\
\hline Management & 1498.60 & $128,879.78$ \\
\hline \multicolumn{3}{|l|}{ Work Sector } \\
\hline Patient care provider & 6208.04 & $2,265,934.82$ \\
\hline Management & 2185.96 & $54,649.09$ \\
\hline Support & 3290.81 & $266,555.62$ \\
\hline \multicolumn{3}{|l|}{ Employment contract } \\
\hline Statutory & 7897.02 & $2,108,505.23$ \\
\hline Civil Servant & 2346.25 & $478,634.30$ \\
\hline \multicolumn{3}{|l|}{ Unit } \\
\hline HUPES & 4990.53 & $1,601,959.03$ \\
\hline CPPHO/AMN & 6567.87 & $985,180.50$ \\
\hline \multicolumn{3}{|l|}{ Work shifts } \\
\hline Day & 5216.27 & $2,206,482.80$ \\
\hline Night & 7930.35 & $380,656.73$ \\
\hline
\end{tabular}

Source: Mean wages in Reais (R\$) throughout the study period (2013). 1 U\$ $=2$ R\$.

The contribution percentage of the first two factorial axes in data total-variability explanation was approximately 25.0\%. According to the other plans generated by FAMC (Figure 2), there was no significant contribution from any of them to the aim of the present study; therefore, we chose to interpret the foreground factorial, only.

The first factorial plan-herein called "perceptual map" (Figure 2)-on the right side of the map shows a group of employees with the following characteristics: absenteeism, women, direct care provider professional category and sector, "statutory workers", working at the Pediatric Center and at the Outpatient Unit, nightshift, sedentary, and HTN negative. This group was named "absenteeism". The "non-absenteeism" group, which is characterized by not being sedentary and smoker, is closer to the center of the factorial axes. The Group of men in the support professional category and sector is on the left side of the perceptual map. The civil servant management professional category and sector is above and to the right of the aforementioned group. It is possible identifying two small groups composed of MS risk factors, obesity and cholesterol, and another one formed by metabolic syndrome and diabetes; both had positive associations. 


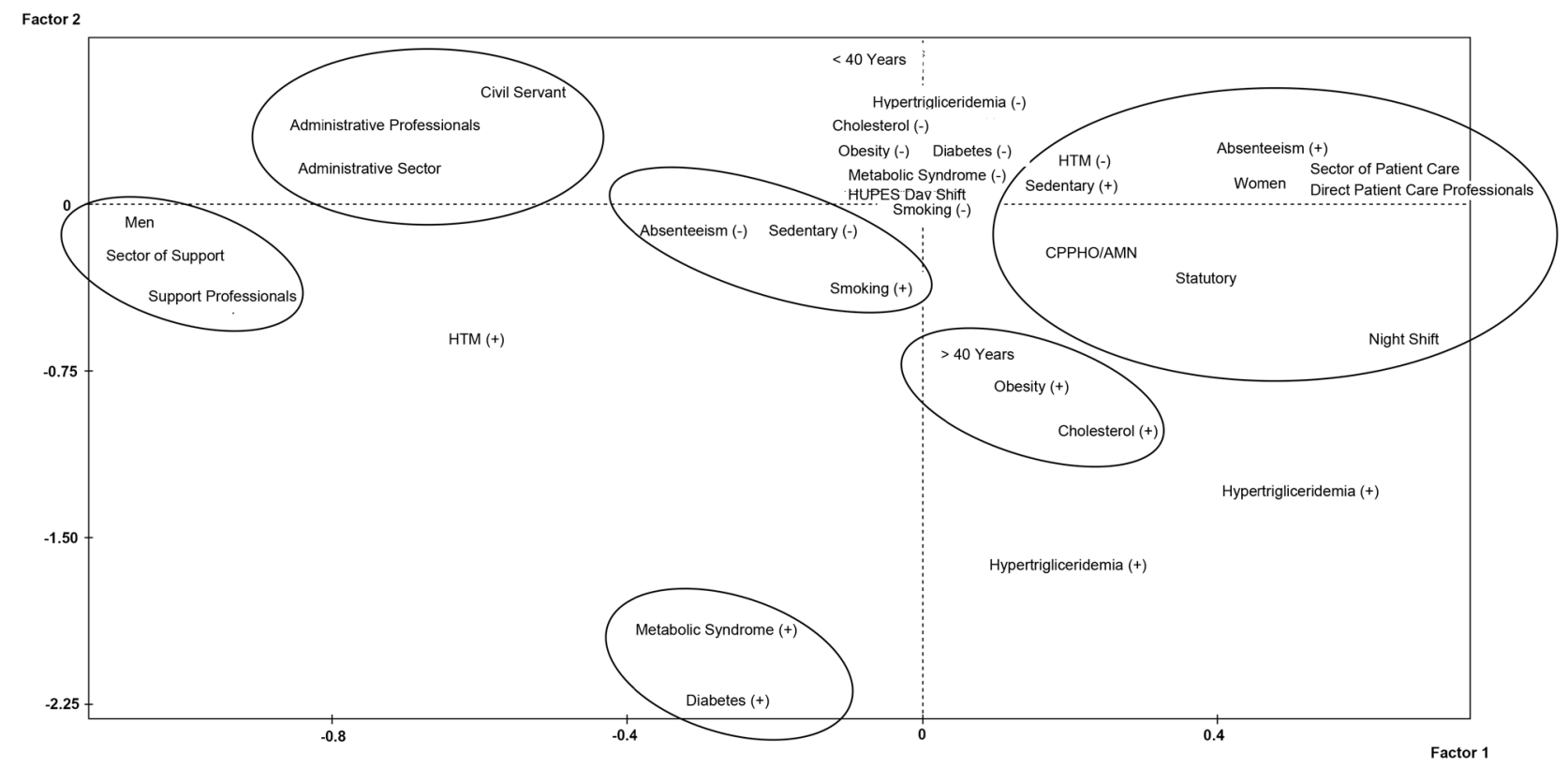

Figure 2. Perceptual map of factorial analysis of multiple correspondences.

Besides being important to stress, the designation "small groups" results from two characteristics that define it. Hypertriglyceridemia and positive HTN do not distinguish one group from another. Dayshift workers from the General Hospital and workers with other MS risk factors are located near the center of the perceptual map in the conjunction of the two factorial axes. Points close to the intersection of the two factorial axes (abscissa and ordinate in the Cartesian plane) did not compose any different grouping. In other words, these features were dilute in other groups.

Table 7 depicts the mean cost per employee according to sex, professional category, activity sector, work unit, employment contract, shift and annual total costs of each category, as well as the total amount of direct costs with absenteeism.

\section{Discussion}

It is consensus that MS prevalence and its risk factors (risk factors for CVD) are increasing worldwide due to age, besides being more predominant in women [1] [16] [27] [28].

Studies about the healthcare professional population are scarce in Brazil, although the MS prevalence in this population is similar to, or even higher than, that of the general population [13] [29] [30].

In addition to the lack of studies in this field, there are no publications on MS prevalence and on its risk factors in healthcare professionals specifying occupational categories, working areas in healthcare institutions, absenteeism prevalence and the impact of absence-related costs. Thus, the present study is pioneer in discussing these topics, fact that makes it difficult to perform comparison. 
Published data, however, show that investigations focused on the health field, mainly on MS or on the prevalence of its individual risk factors, restricted this population to certain occupational categories, namely: physicians [31], nurses only [12], physicians, nurses and paramedics [32], and occupational categories concerning patient care providers, management and support professionals [29].

It is worth pointing out that the workplace, mainly healthcare institutions, represents one of the most promising scenarios for early disease detection and intervention monitoring [1]. Such result corroborates the recommendation for using MS diagnoses to identify patients with high cardiovascular complication risks and to establish a preventive approach to prolong life in the adult population [27].

International Studies on healthcare professionals have shown MS prevalence similar to, and higher than, that found in the present study: 76 (6.6\%). Rates recorded in other countries were: $4.4 \%$ in Chile [32], 5.7\% in Italy [33], 7.8\% in Spain [28], 7.9\% in Turkey [3], 9.5\% in Thailand [34], 23.8\% in Egypt [31], $24.3 \%$ in Malaysia [12], and 34\% in Africa [13].

It was observed that MS prevalence was far below in the current study than in other two studies conducted in Porto Alegre-Rio Grande do Sul State in institutions showing similar characteristics to the herein assessed institution, although with smaller populations. The first study conducted at Hospital de Clínicas de Porto Alegre showed 20\% ( $n=290)$ MS prevalence, even when obese patients were separately assessed; the prevalence was similar $(24.6 \%)$ to that of the general population (20\% to $25 \%$ ) [30]. The second study carried out at Hospital Complex of Santa Casa de Porto Alegre revealed 12.8\% ( $n=740)$ MS prevalence [29]. The MS prevalence in the present study was higher in women, and it increased with age (40 years); it was similar between professional categories such as patient care provider and support, as shown in the literature [29] [30] [31] [34].

The high prevalence of diseases associated with MS inpatient care providers stands out. Employees in charge of office and management support ensure that the provided work procedures assure proper patient care. UH/UFBA women, nursing professionals and management assistants showed the highest MS and MS risk factor prevalence, including physical inactivity and smoking.

Age and educational level are well defined factors associated with MS in the literature [3] [5] [29]. This information complies with the present findings, which showed higher MS prevalence in mid-level training employees.

Studies show that the low educational levels and wages in the general population are related to MS in the female population, likely because women from higher socioeconomic status tend to be more attentive to fitness, to healthy eating and to exercising [5].

The higher HTN prevalence recorded for the healthcare professional population was $20.4 \%$ in Taiwan [35]. Countries such as Chile (31.1\%) [32] and Spain (20.3\%) had results similar to the present ones [28]. A study conducted at Albert Einstein Hospital, São Paulo-Brazil, reported 18.9\% [6] MS prevalence and 
another one performed in Bahia had 24.9\% MS prevalence [36], similar to the current one (25\%). Special attention should be given to hypertension, since it was the most prevalent disease in the current study, as well as in another international study [28]. The present study depicts a clear association between HTN, MS, HTN, and professional category (p-value $=0.0001$ ). There is controversy, however, about the association between occupational status and HTN prevalence. Workers are more specialized in patient care categories in healthcare institutions; these data corroborate the literature and show higher HTN prevalence in non-specialized and lower wage workers [2]. Similarly, the herein evaluated hypertensive population showed the highest MS prevalence in mid-level training workers and in the support category.

There are also references about the correlation between HTN, and other risk factors, with occupational stress, smoking, chemical and physical hazards, satisfaction with work, occupation, lifestyle, weight, exposure to noise, work per shift, decreased productivity and life expectancy, increased absenteeism and costs for the healthcare system [1] [2] [10] [14] [16] [25] [27] [28] [29] [37] [38].

The HTN self-reported by health professionals in the present study corresponded to 233 (20.3\%), nevertheless 284 (25\%) hypertensive subjects were identified. Such result evidences that, although a significant percentage of employees are hypertensive, little attention is given to the problem, and it justifies the importance of this monitoring type, even in populations known to be more informed about the herein addressed topic.

With respect to overweight, data showed 634 cases $(54.0 \%)$ in comparison to other studies, such as that conducted with Mexican nurses (54.9\%) [5], nursing workers, management workers, African industrial and health attendants (56.0\%) [13], and Brazilian health workers (50.1\%) [29]. These numbers are worrisome, since overweight is a risk factor for HTN development [6], to DMT2 and hypertriglyceridemia [35] and consequent CVD, affecting the productivity and quality of life [1]. Overweight and obesity have shown higher prevalence in mid-level, support and patient care provider employees.

Obesity is the risk factor that most significantly contributes to MS [1] [30]. It affects direct care providers and impacts service provision to users due to worker absence caused by sick leave.

The present data on the prevalence of obese employees show larger numbers than studies conducted in Turkey and in Spain, which recorded $13.8 \%$ and $12.5 \%$ frequency in health care professionals, respectively [3] [28]. Results were similar to those found in Egypt (18.7\%) and lower than those revealed in Taiwan (24.7\%) [36] and Pakistan (28.2\%) [39]. Previous Brazilian studies showed 16.6\% MS prevalence in Porto Alegre [29], 17.7\% in Piaú [37] and 16\% in Bahia [40].

The association between obesity, HTN, DM, MS and sex and, night shift was statistically significant.

The association among MS risk factors, work shift and nightshift [38] [41] [42] [43] in institutions providing patient care [6], mainly in the nursing staff, has also been demonstrated [33] [42]. Another aspect to be taken into considera- 
tion is that the nightshift may be responsible for emotional and psychological issues capable of triggering absenteeism causes [14]. With regard to the present study, $21.6 \%$ of the workers who carry out their activities in the nightshift recorded absenteeism due to osteoarticular diseases.

Diabetes mellitus and HTN are the main CVD risk factors, besides social inclusion undertaking, early retirement and high morbidity and mortality rates [44]. Although diabetes was the least prevalent risk factor in the present study, 36 employees (49.3\%) with diabetes had MS, showing a significant association ( $p$-value $=0.0001)$. Moreover, the present findings showed apparent blood sugar control in healthcare professionals. Likely, the effective work done by the outpatient and Diabetes group of HU/UFBA has influenced the results.

With respect to physical activity, $756(64.4 \%)$ employees were sedentary and only $59(5.1 \%)$ of them were smokers. The percentage of sedentary individuals among adults in Brazil corresponds to 77\% [45].

The prevalence of smoker employees was much lower than that in the general Brazilian population; a study conducted in a similar population in Spain rated $18.3 \%$ [28]. This result can certainly be attributed to strong anti-tobacco campaigns and to the creation of the tobacco control clinic by the Pneumology Service at HU/UFBA.

The complexity characterizing hospitals, particularly hospitals focused on teaching, demands commitment to the health of its employees. All MS and CVD risk factor occurrences in employees directly influence the management procedures in health institutions [18], due to increased cost [1], decreased productivity [1], to the influence on the quality of services provided to users ${ }^{42}$ and to high absenteeism rates [1].

The awareness about the absenteeism rate and its main determinants in an important tool to manage the Organization and its employees. It works as management information for the elaboration of prevention and rehabilitation programs focused on healthcare professionals.

With respect to absenteeism, is evident in the current study, that absenteeism was much higher (40.1\%) than the expected by the International Labor Organization (ILO) - (2.5\%) [17]. A study conducted in the UH of São Paulo University recorded $8.7 \%$ [21] absenteeism prevalence, and this result was similar to that recorded in a study performed in Africa, 47.9\% [46]; however, it was lower than that recorded in a UH in Paraná State (72.6\%) [15]. The higher values found in the here in mentioned studies can be justified by the study populations, which were exclusively constituted by the nursing staff [14] [15] [16] [21]; different from the present study, which presented absenteeism prevalence in all professional categories in the institution.

The difference in the numbers of days out of work per employment contract recorded for the herein assessed population may be associated with the characteristic of the Brazilian public service, whose employees feel highly protected by employment schemes; which are also found in public and university organizations [21]. 
The larger number of days out of work was presented by patient care providers such as nursing professionals, who were the most absent due to sick leave, 253 (54.5\%). This number is far above the current 6\% estimated by the Federal Council of Nursing Care (COFEN) [47]. Management assistants showed 53 (11.3\%) absence prevalence, and they were followed by kitchen assistants, 21 (4.5\%). However, there is no comparative benchmark to other professional categories.

When the most prevalent issues are analyzed in light of the employment relations, small age differences do not seem to be a factor-modifier, since risk factors commonly associated with absenteeism could not be demonstrated.

The high absenteeism rate recorded for nursing professionals can be associated with the work process itself, because it requires physical exertion, repetitive activities, nightshift schedules and the need of dealing with losses and with suffering [14] [16]. Osteoarticular diseases significantly contributed to absenteeism, and it corroborates the literature [14] [18] [21] [43].

It is worth noticing that employees away from work due to Osteoarticular diseases, 84 (62.6\%), were overweight; of those, 36 (42.9\%) were obese and 48 (57.1\%) were overweight.

The lack of association between risk factors and scientifically correlated diseases stands out in the present study possibly because of underreporting or of other motivations for sick leave other than the herein reported subjective complaints.

The impact generated by absenteeism on public healthcare institutions does not differ from that caused on other organizations, mainly because it influences the health of the assessed population and generates public spending. When it comes to the healthcare field, it is worth analyzing the multidisciplinary features, the need of complete working teams depending on the service, occupational categories and work shift; therefore, there can be no human labor replacement.

The present results evidenced that female 'statutory' employees belonging to the healthcare provider category working at HUPES in the nightshift were responsible for the highest mean costs with absenteeism caused by sick leave. The mean cost with absenteeism over the studied period among HU/UFBA employees was $\mathrm{R} \$ 5492.87$, and it represents $\mathrm{R} \$ 2,587,139.53$ in total (Table 7).

The annual HU/UFBA budget in the studied period totaled $\mathrm{R} \$ 40,421,652.55$. Absenteeism represented 6.4\% financial impact on the annual budget. However, if only the resources intended for funding current costs are taken into account, this percentage rises to $17 \%$, fact that reflects the important repercussion of it on production financing.

If one acknowledges the financial impairments faced by University Hospitals over the years, these financial resources could be invested in improving processes and working conditions, workers' training and leadership, technology, besides others aspects directly influencing illness cases among employees.

Therefore, the prevention of MS risk factors in the workplace should be implemented by promoting health programs focused on the institution's em- 
ployees. These programs deserve substantial reinforcement in healthcare institutions, mainly in activities performed by direct care providers, in order to detect illnesses related to the metabolic syndrome.

Health promotion in the workplace can be seen as a benefit to employees, to the institution and to productive systems. Teaching Hospitals benefit from absenteeism reduction, since it decreases productivity costs from health service promotion and from knowledge production to students and teachers. It contributes to the institution's efficient and effective management space and to fulfilment of its mission.

\section{Conclusions}

- This is the first study describing the prevalence of MS risk factors and the absenteeism profile of hospital workers, as well as the impact of costs with absenteeism caused by sick leave among workers in all occupational categories, activity sectors, work shifts and occupational ties.

- The prevalence of MS risk factors in the studied population was $25.0 \%$ hypertension, $20.0 \%$ hypercholesterolemia, $18.4 \%$ obesity, $9.9 \%$ hypertriglyceridemia, 6.5\% DM, and 6.6\% MS itself.

- Overweight was recorded for 634 (54\%) employees. There was statistically significant association between obesity and nightshift ( $\mathrm{p}$-value $=0.000)$; it points out that $47(74.6 \%)$ of direct care providers working in the nightshift were overweight.

- Women, nursing professionals and management assistants showed the highest prevalence of MS and of its risk factors, including physical inactivity and smoking.

- Absenteeism accounted for $40.1 \%$ of the sample; the greatest absenteeism prevalence was found in employees with obesity (PR 1.38, CI: $-1.811 .05)$ and hypercholesterolemia (PR: 1.56, CI: -2.29 1.06).

- Female employees working in categories and sectors such as patient care provider, "statutory", sedentary, obese, and employees with hypercholesterolemia and those working in the Pediatric Center and in the Outpatient unit had association with absenteeism.

- Female employees, "statutory" working with patient care giving at HUPES in the nightshift were responsible for the highest mean costs with absenteeism caused by sick leave.

- The mean annual cost with absenteeism among HU/UFBA employees was $\mathrm{R} \$ 5492.87$, and it represents $\mathrm{R} \$ 2,587,139.53$, number that significantly reduces the workers' productive capacity.

- Special attention should be given to nursing technicians and management assistants, who presented the highest MS, Obesity, Dyslipidemia, HTN, DM, sedentariness, absenteeism and sick leave prevalence rates due to osteoarticular diseases.

The challenge for managers in University Hospitals lies on financing and on successful managerial practices, but also on the systematic and in-depth look at 
illness processes presented by the employees, on the resignification of work processes in healthcare and on the institutionalization of health promotion policies focused on healthcare professionals.

\section{Final Considerations}

Regardless of the criteria used to define MS, the concern with each risk factor is consensus, mainly in healthcare institutions, even if the employees present at least one risk factor. Such result should be a warning and encourage the implementation of actions aiming at decreasing repercussions affecting quality of life and labor productivity to assure the delivery of health services to the population.

By Understanding that obesity is a MS and CVD risk factor, and since it is the easiest clinical manifestation to be diagnosed, special attention should be given to the implementation of weight control and comorbidity prevention strategies.

Results showed significant productive capacity and, consequently, reduced financial resources in a Hospital focused on public education and on assisting Public Health System users. In addition to the social cost, emphasis should be given to the individual cost of each employee who got sick due to suffering, discouragement and emotional distress.

When it comes to public institutions, managers must suggest higher instances of Government actions, which should be incorporated to health policies focused on the workers, as well as fight for better features and working conditions.

Accordingly, the management of occupational health, even of healthy individuals, as well as the identification of prevalent risk factors and the adoption of actions and strategies to minimize absenteeism due to sick leave to promote quality of life and to assure quality and safety to users must be the responsibility of managers.

According to the present results, the herein assessed UH should review its work processes and invest in institutional communication areas (educational guidelines on health, quality of life, healthy nutrition, ergonomics, among others). Activities such as computerized systems for cost management; psychological and behavioral care workers; preventive maintenance in the equipment-mainly in closed units such as ICU, Surgical Center (SC), laundry and nutrition-, mid-level managers' training on how to deal with workers' skills (mainly with the sick and absent ones), absenteeism and presenteeism monitoring, and dealing with conflicts; gymnastics promotion; strengthening occupational health services, health clinics and Host Program to workers towards self-care, motivational activities and social integration (coral, ballroom dancing, theater, breakfast and holiday parties) and encouragement for sports should be adopted.

Our findings showed that, in addition to social and personal issues, the definition of work shifts should indicate the benefits to the health of healthcare workers, mainly those with already diagnosed risk factors, who should follow rotating scales and different shifts. These actions can be an important tool for personnel 
management teams in complex organizations such as University Hospitals.

The promising scenario that emerges from UHs in Brazil must assure the conditions for adequate process people and result management; and, consequently, rescue the image of University Hospitals.

\section{References}

[1] Leutzinger, J., Pirner, M., Landschulz, W., Nevins, R.L., Sullivan, S., Silberman, C., et al. (2006) Metabolic Syndrome and Worksite Health Promotion. American Journal of Health Promotion, 21, 3.

[2] Cordeiro, R., Fischer, F.M., Filho, E.C.L. and Filho, D.C.M. (1993) Ocupação e hipertensão. Revista de Saúde Pública, 27, 380-387. https://doi.org/10.1590/S0034-89101993000500010

[3] Oğuz, A., Sağun, G., Uzunlulu, M., Alpaslan, B., Yorulmaz, E., Tekiner, E., et al. (2008) Frequency of Abdominal Obesity and Metabolic Syndrome in Healthcare Workers and Their Awareness Levels about These Entities. Turk Kardiyoloji Dernegi Arsivi, 36, 302-309.

[4] Alegría, E., Cordero, A., Laclaustra, M., Grima, A., León, M., Casasnovas, J.A., et al. (2005) Prevalence of Metabolic Syndrome in the Spanish Working Population: MESYAS Registry. Revista Española de Cardiología, 58, 797-806. https://doi.org/10.1157/13077231

[5] Sanchez-Chaparro, M.A., Calvo-Bonacho, E., González-Quintela, A., FernándezLabandera, C., Cabrera, M., Sáinz, J.C., et al. (2008) Occupation-Related Differences in the Prevalence of Metabolic Syndrome. Diabetes Care, 31, 1884-1885. https://doi.org/10.2337/dc08-0431

[6] Sarno, F. (2005) A importância do excesso de peso e da obesidade na determinação da hipertensão arterial sistêmica em adultos em uma população de funcionários de um hospital de grande porte de São Paulo. Masters Degree Dissertation, Universidade São Paulo, São Paulo.

[7] Schultz, A.B. and Edington, D.W. (2009) Metabolic Syndrome in a Workplace: Prevalence, Co-Morbidities, and Economic Impact. Metabolic Syndrome and Related Disorders, 7, 459-468. https://doi.org/10.1089/met.2009.0008

[8] Goetzel, R.Z., Gibson, T.B., Short, M.E., Chu, B.-C., Waddell, J., Bowen, J., et al. (2010) A Multi-Worksite Analysis of the Relationships among Body Mass Index, Medical Utilization, and Worker Productivity. Journal of Occupational and Environmental Medicine, 52, S52-S8. https://doi.org/10.1097/JOM.0b013e3181c95b84

[9] Jans, M.P., Van den Heuvel, S.G., Hildebrandt, V.H. and Bongers, P.M. (2007) Overweight and Obesity as Predictors of Absenteeism in the Working Population of the Netherlands. Journal of Occupational and Environmental Medicine, 49, 975-980. https://doi.org/10.1097/JOM.0b013e31814b2eb7

[10] Tachang, G.K., Choukem, S.P., Ndjebet, J., Dzudie, A. and Titanji, V.P.K. (2012) Prevalence of Hyperglycaemia, Obesity and Metabolic Syndrome (a Three Component Study) among Hospital Personnel in the Littoral Region of Cameroon. International Journal of Medicine and Medical Sciences, 4, 232-237.

[11] Marziale, M.H.P. (1995) Condições ergonômicas da situação de trabalho, do pessoal de enfermagem, em uma unidade de internação hospitalar. Ribeirão Preto, $\mathrm{PhD}$ Dissertation, Escola de Enfermagem de Ribeirão Preto, Universidade de São Paulo, São Paulo, 155 p.

[12] Ahmad, F.A., Mohd, N.S. and Wan, M.Z.W.M. (2011) Metabolic Syndrome and 
Occupational Risk Factors among Healthcare Workers in Kelantan. Health and the Environment Journal, 2, 23-28.

[13] Garrido, R.A., Semeraro, M.B., Temesgen, S.M. and Simi, M.R. (2009)Metabolic Syndrome and Obesity among Workers at Kanye Seventh-Day Adventist Hospital, Botswana. South African Medical Journal, 99, 331-334.

[14] Costa, F.M., Vieira, M.A. and Sena, R.R. (2009) Absenteísmo relacionado à doenças entre membros da equipe de enfermagem de um hospital escola. Revista Brasileira de Enfermagem, 62, 38-44. https://doi.org/10.1590/S0034-71672009000100006

[15] Silva, D.M.P.P. and Marziale, M.H.P. (2000) Absenteísmo de trabalhadores de enfermagem em um Hospital Universitário. Revista Latino-Americana De Enfermagem, 8, 44-51. https://doi.org/10.1590/S0104-11692000000500007

[16] Sancinetti, T.R., Soares, A.V.N., Lima, A.F.C., Santos, N.C., Melleiro, M.M., Fugulin, F.M.T., et al. (2011) Taxa de absenteísmo da equipe de enfermagem como indicador de gestão de pessoas. Revista Da Escola De Enfermagem Da USP, 45, 1007-1012. https://doi.org/10.1590/S0080-62342011000400031

[17] Organizacion International del trabajo (IOT) (1989) Absenteismo: Causas y control. In: Enciclopedia de Salud y Seguridade en el Trabajo, Madrid, Vol. 1, 5-12.

[18] Aguiar, G.A.S. and Oliveira, J.R. (2009) Absenteísmo: Suas principais causas e consequências em uma empresa do ramo de saúde. Revista de Ciências Gerenciais, 13, 95-113.

[19] Chiavenato, I. (2000) Recursos Humanos. Atlas, São Paulo.

[20] Berrocal, I., Jiménez, M., Rojas, J.C. and Salazar, N. (2012) Índices Y Estadísticas Del Absenteismo Laboral. Caso Universidad Nacional. Revista de la Faculdad de Ciencias Sociales Universidad Nacional, 32, 1-20.

[21] Reis, R.J., La Rocca, P.F., Silveira, A.M., Bonilla, I.M.L., Gine, N.A. and Matín, M. (2003) Fatores relacionados ao absenteísmo por doenças em profissionais de enfermagem. Revista De Saude Publica, 37, 616-623. https://doi.org/10.1590/S0034-89102003000500011

[22] Andrade, T.B., Souza, M.G.C., Simões, M.P. and Andrade, F.B. (2008) Prevalência de absenteísmo entre trabalhadores do serviço público. Scientia Medica, 18, 166-171.

[23] WHO (1995) Physical Status: The Use and Interpretation of Antropometr. Report of a WHO Expert Committee, 439, Geneva.

[24] International Diabetes Federation (2005) The IDF Consensus Worldwide Definition of the Metabolic Syndrome.

https://www.idf.org/our-activities/advocacy-awareness/resources-and-tools/60:idfco nsensus-worldwide-definitionof-the-metabolic-syndrome.html

[25] Organização Mundial da Saúde. Centro Colaborador da OMS para a Classificação de Doenças em Português. Classificação Estatística Internacional de Doenças e Problemas Relacionados à Saúde (1996) 10ª Revisão. EDUSP, 3 ed. São Paulo. http://www.datasus.gov.br/cid10/V2008/cid10.htm

[26] Greenacre, M.J. (1981) Practical Correspondence Analyses. In: Barnett, V., Ed., Interpreting Multivariate Data, Wiley, Chichester, 119-146.

[27] Mottillo, S., Filion, K.B., Genest, J., Joseph, L., Pilote, L., Poirier, P., et al. (2010) The Metabolic Syndrome and Cardiovascular Risk: A Systematic Review and Meta-Analysis. Journal of the American College of Cardiology, 56, 1113-1132. https://doi.org/10.1016/j.jacc.2010.05.034

[28] Guisado, J.G., Manzano, J.J.L., Cid, J.R., Segura, C.G. and Rufach, M.T.L. (2008) 
Prevalencia de síndrome metabólico em populación laboral. El corazón de Asepeyo. Annales De Medecine Interne, 25, 325-330.

[29] Basei Rossa, C.E., et al. (2012) Síndrome metabólica em trabalhadores de um hospital universitário. Revista Portuguesa de Cardiologia, 31, 629-636.

https://doi.org/10.1016/j.repc.2012.07.002

[30] Rodrigues, T.C., Winter, J.J., Trindade, D.M. and Canani, L.H.S. (2007) Prevalência de Síndrome Metabólica em uma população de pacientes com excesso de peso (funcionários de um hospital de referência). Rev HCPA, 27-30.

[31] Almikhafy, A.A., Maklady, F.A., Sobhy, S.A., Elsheikh, E.I. and Alansi, A.K. (2008) Metabolic Syndrome among Yemeni Psysicians in Sana'a. Suez Canal University Medical Journal, 11, 31-34.

[32] Díaz, C.M., Novik, A.V., Oyaneder, S.R., Hinojosa, O.C. and Tobar, R.A. (2007) Prevalencia de síndrome metabólico en funcionarios del servicio de medicina interna del hospital Dr. Gustavo Fricke Viña del Mar. Boletín del Hospital de Viña del Mar, 63, 101-109.

[33] Pietroiusti, A., Neri, A., Somma, G., Coppeta, L., Iavicoli, I., Bergamaschi, A., et al. (2010) Incidence of Metabolic Syndrome among Night-Shift Healthcare Workers. Occupational and Environmental Medicine, 67, 54-57. https://doi.org/10.1136/oem.2009.046797

[34] Phengtham, U. (2011) Prevalence of Syndrome among Health Care Personnel and Office Workers in Lampang Hospital. Lampang Medical Journal, 32, 11-17.

[35] Hwang, L.C., Tsai, C.H. and Chen, T.H.H. (2006) Overweight and Obesity-Related Metabolic Disorders in Hospital Employees. Journal of the Formosan Medical Association, 105, 56-63. https://doi.org/10.1016/S0929-6646(09)60109-1

[36] Aquino, E.M.L., Araújo, M.J.S., Menezes, G.M.S. and Marinho, L.F.B. (1993) Saúde e trabalho de mulheres profissionais de enfermagem em um hospital público de Salvador, Bahia. Revista Brasileira de Enfermagem, 46, 245-247. https://doi.org/10.1590/S0034-71671993000300007

[37] Sousa, R.M.R.P., Sobral, D.P., Paz, S.M.R.S. and Martins, M.C.C. (2007) Prevalência de sobrepeso e obesidade entre funcionários plantonistas de unidades de saúde de Teresina, Piauí. The Revista de Nutrição, 20, 473-482. https://doi.org/10.1590/S1415-52732007000500003

[38] Biggi, N., Consonni, D., Galluzzo, V., Sogliani, M. and Costa, G. (2008) Metabolic Syndrome in Permanent Night Workers. Chronobiology International, 25, 443-454. https://doi.org/10.1080/07420520802114193

[39] Mahmood, S., Kazim, M.R.N., Ali, N., Tousuf, N. and Hamid, Y. (2010) Predictors of Obesity among Post Graduate Trainee Doctors Working in a Tertiary Care Hospital of Public Sector in Karachi, Pakistan. Journal of the Pakistan Medical Association, 60, 758-761.

[40] Aquino, E.M.M.L.L., Magalhaes, L.B.N.C., Araújo, M.J., Almeida, M.C.C. and Leto, J.P. (2001) Hipertensão arterial em trabalhadoras de enfermagem: Padrão de ocorrência, diagnóstico e tratamento. Arquivos Brasileiros De Cardiologia, 76, 197-202.

[41] Knutsson, A., Akerstedt, T. and Jonsson, B.G. (1988) Prevalence of Risk Factors for Coronary Artery Disease among Day and Shift Workers. Scandinavian Journal of Work, Environment \& Health, 14, 317-321. https://doi.org/10.5271/sjweh.1913

[42] Mina, H.A. and Jungsun, P. (2005) Shiftwork and Metabolic Risk Factors os Cardiovascular Disease. Journal of Occupational Health, 47, 89-95.

https://doi.org/10.1539/joh.47.89 
[43] Karlsson, B., Knutsson, A. and Lindahl, B. (2001) Is There an Association between Shift Work and Having a Metabolic Syndrome? Results from a Population Based Study of 27,485 People. Occupational and Environmental Medicine, 58, 747-752. https://doi.org/10.1136/oem.58.11.747

[44] Sociedade Brasileira de Diabetes (SBD) (2009) Diretrizes da Sociedade Brasileira de Diabetes. SBD, São Paulo.

[45] Brasil. Ministério da Saúde. Pesquisa de orçamentos familiares (POF) 2008-2009 (2010) Antropometria e estado nutricional de crianças, adolescentes e adultos no Brasil. Rio de Janeiro.

[46] Uganda National Health Users'/Consumers' Organization (UNHCO) (2002) Survey to Establish the General Level of Awareness about Patients' Rights. UNHCO, Kampala.

[47] Conselho Federal de Enfermagem (COFEN) Resolução n. 293/04, de 21 de setembro de 2004. http://www.cofen.gov.br/resoluo-cofen-2932004_4329.html 